\title{
LAAS GEEL (SOMALILAND): 5000 YEAR-OLD PAINTINGS CAPTURED IN 3D
}

\author{
L.Grenier'P.Antoniotti , G.Hamon, D.Happe \\ ${ }^{a}$ Art Graphique et Patrimoine - 15, 15bis Av. J. Jaurès - 94340 Joinville-le-Pont - FRANCE
}

KEY WORDS: Laas Geel, Archaeology, Somaliland, Photogrammetry, 3D-modeling, laser-scanning.

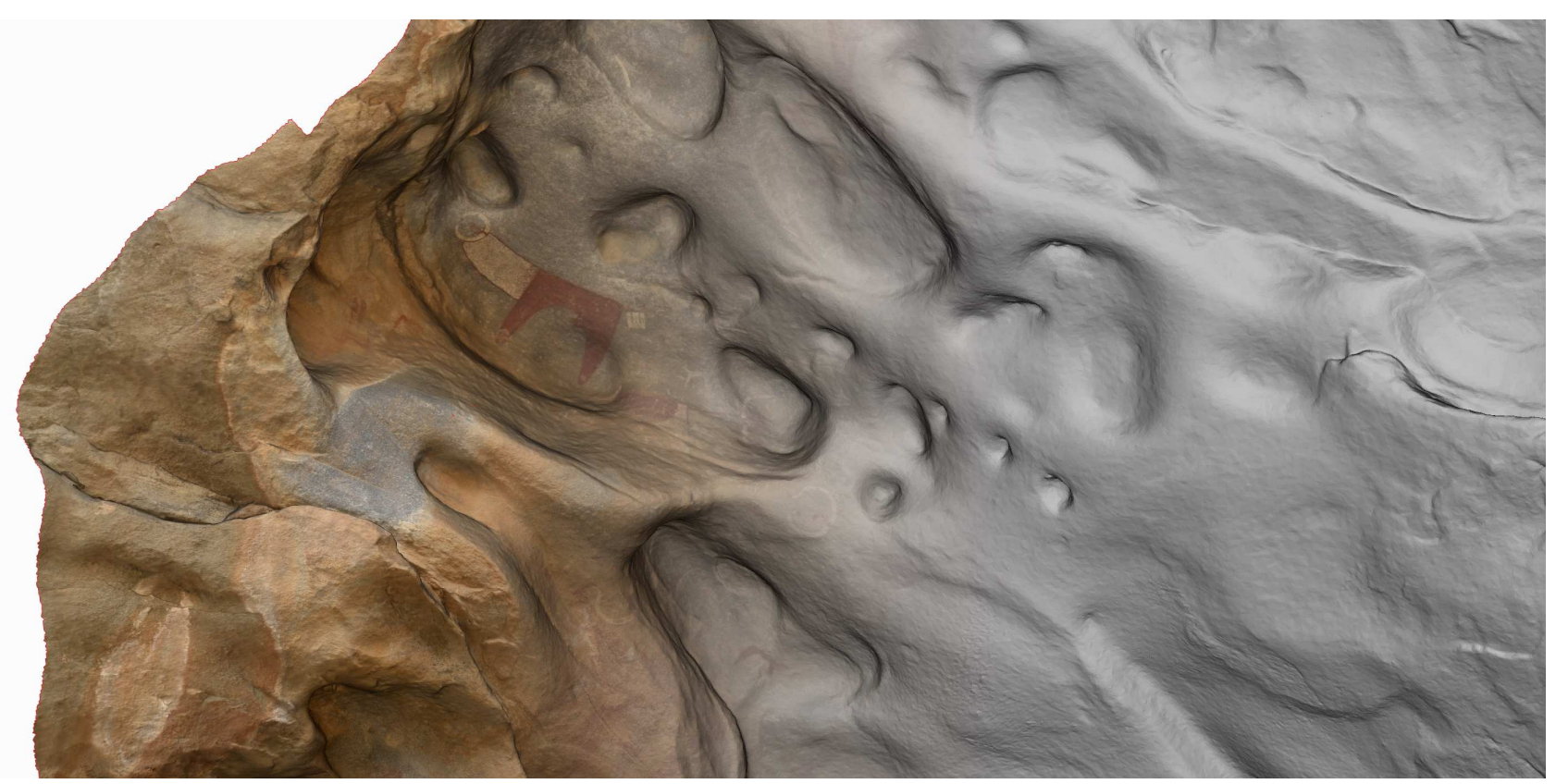

ABSTRACT

Discovered in 2002 by a French archaeology team conducted by Prof. X. Gutherz, Laas Geel (Somaliland), is probably one of the most remarkable archaeological site in the horn of Africa. Located in an isolated arid region, it is made of natural rocky shelters on which hundreds of colored paintings still remain in a particularly good state of conservation. The first studies achieved in the last decade let suppose that they are 5000 years old. After several studying and exploring expeditions, a 3Ddigitizing campaign has been carried out by Art Graphique et Patrimoine, under the direction of X. Gutherz, with the support of the cultural service of the French Embassy in Djibouti. The project was focused on three main goals: production of a high accuracy 3D-documentation for scientific needs, archiving the 3D digital print recorded on site for the conservation and the saving of this heritage, and finally diffusing the results throughout various kinds of media to reveal the site to the public, insisting on its vulnerability.

\section{GENERAL OVERVIEW OF THE PROJECT}

\subsection{Geopolitical context}

The Laas Geel site is located in the Republic of Somaliland. Not recognized by the international community, it officially belongs to Somalia, but enjoys a de facto independence since 1991, when the region seceded. Laas Geel is located halfway between the two main cities of the country, Hargeisa and Berbera.

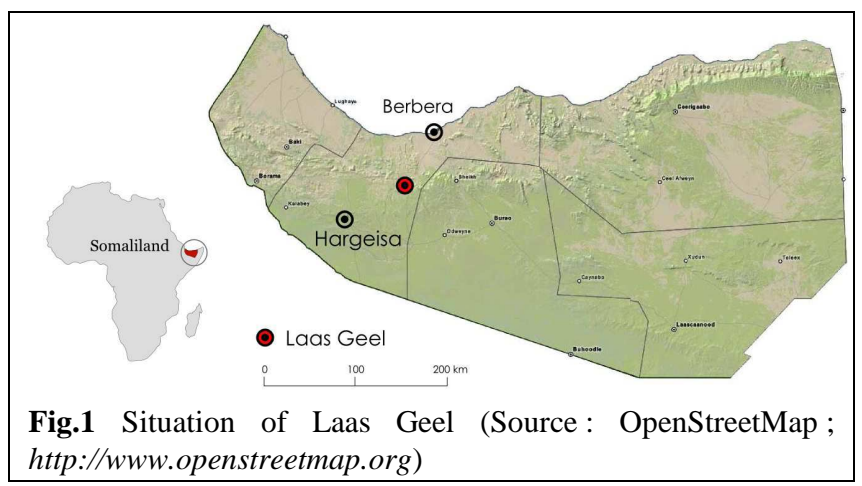




\subsection{Site presentation}

Laas Geel (literally "water point for camels") is located at the confluence of two often dry rivers, on the slopes of a granite rocky hill overlooking an arid shelf between 800 and 900 meters above sea level.

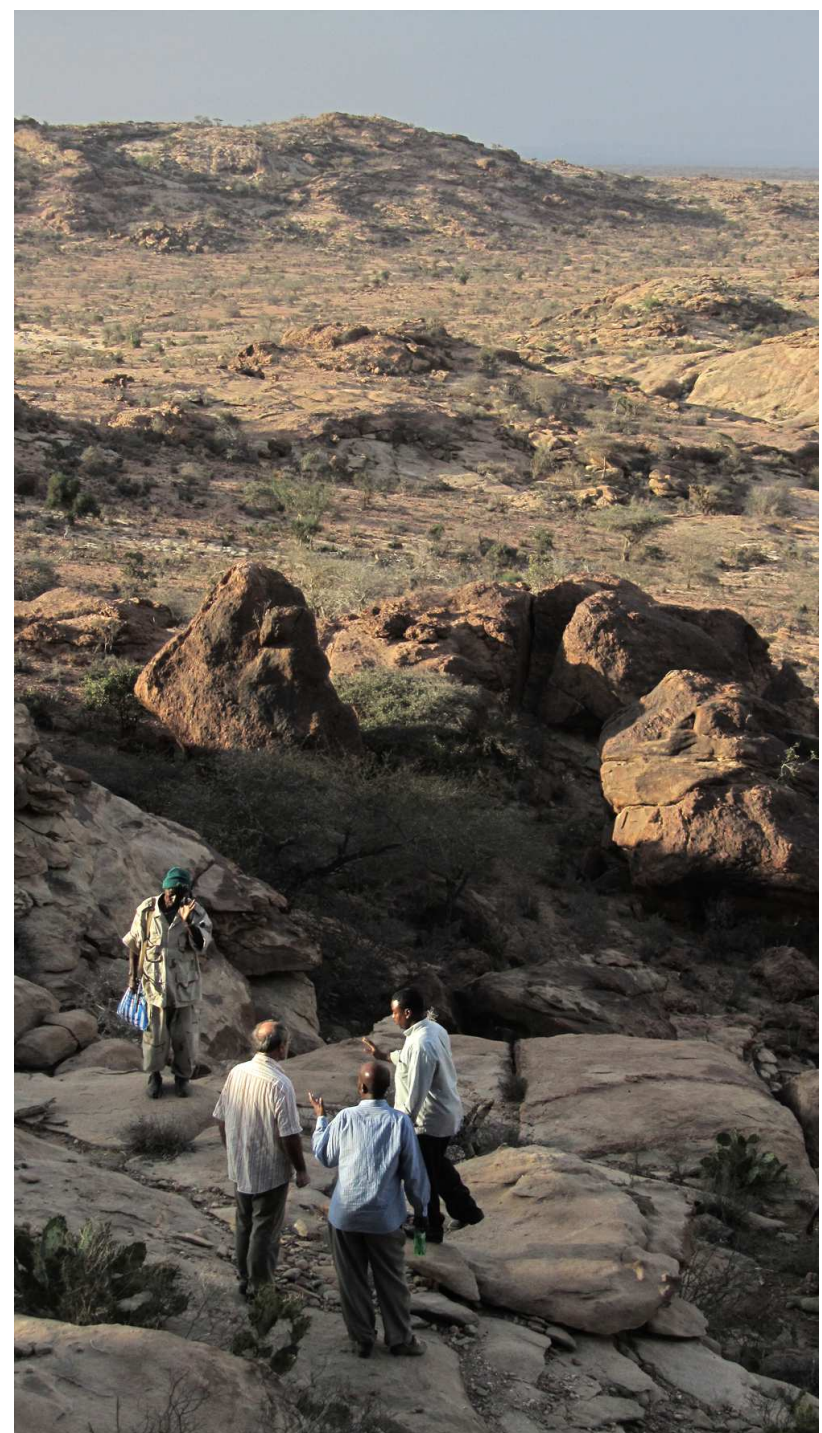

Above: first visit in Laas Geel. Below: Colored paintings of shelter nb.1

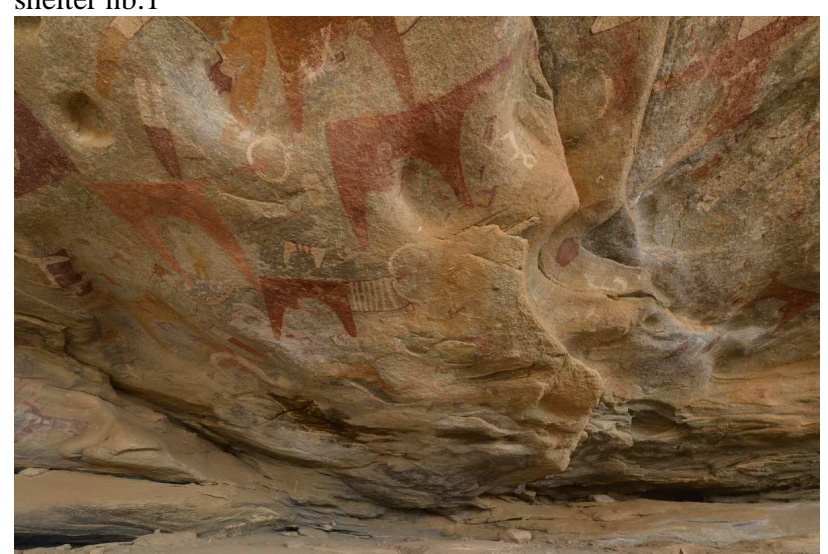

22 shelters have been listed, spread on a 10 hectares area, for a size varying between 10 and more than 200 square meters. They are made of smooth walls, protected from erosion by overhangs, explaining the excellent state of conservation of the paintings.

The different representations, almost related to rearing sceneries (especially cows), remind also human outlines and other wild animals. However, these paintings still raise many questions on which archaeologists keep working on, and this is one of the main purpose of the survey campaign.

\subsection{Purpose of the operation}

Several archaeological studies have been achieved in Laas Geel in the last five years, raising the importance of a 3D accurate and exhaustive survey. The mission thereby was focused on three main goals :

- High-resolution 3D-digitalisation and exhaustive photogrammetric acquisitions on the most remarkables shelters, in order to provide interactive three-dimensional models and high-resolution ortho-photos with colorimetric control. A medium-resolution digitalization will be performed in the same time on the other shelters.

- Very high-resolution 3D-digitalisation on the most important areas, with a sub-millimetric accuracy, in order to reveal the most tiny details.

- Topographic survey, allowing a global georeferencing of the data.

\section{METHODOLOGICAL CHOICES AND PREPARATION}

Methodological choices and the ensuing survey operations have been influenced by several constraints and opportunities that finally lead us to use mainly photogrammetry in most of the cases. The first reason for this choice is the need to produce three-dimensional models with the best possible quality textures, in order to make the results exploitable. Moreover, the last software improvements in automatic points detection now allow to generate accurate and dense photomodels, in a very short time.

Combined to photogrammetric acquisitions, a 3Dlaserscanning survey was carried out for each shelter of the site, in order to get a spare 3D databank in case of possible problems in the photogrammetric process.

Finally, the global georeferencing of the data, was performed by a topographic classical survey: setup of a polygonal canvas, and recording of survey points (natural points on the paintings, in order to avoid masking areas with targets).

\section{THE SURVEY MISSION}

\subsection{Meeting authorities and first visit on the site}

After a short meeting with officials of the French Embassy in Djibouti, the team flew to Hargeisa, where an official visit was organized at the Ministry of Commerce, Industry and Tourism, in order to present to the Minister, M. Abdirizak Khalif Ahmed the workflow, as well as enhancing the partnership previously established between the different 
stakeholders, including the distribution of a booklet of fifteen pages presenting the site and the work that had been carried out during the previous missions. In the afternoon, a first visit of the site was organized in order to evaluate the amount of work and validate the workflow.

\subsection{Laserscanning operations}

The first step of the survey operations was the 3D-recording of the most remarkable shelters. This work was quite fast, thanks to the high speed laserscanners that were used, but also to the good visibility of each zones of the shelters, allowing an exhaustive survey with only few scan stations. Spatial resolution of the scanner was set to $6 \mathrm{~mm}$ at $10 \mathrm{~m}$. Given the distance between scanner and paintings, often much shorter, this setting provided point clouds with a spatial resolution most of the time lower than $3 \mathrm{~mm}$, which is far beyond the requested information on the most areas.

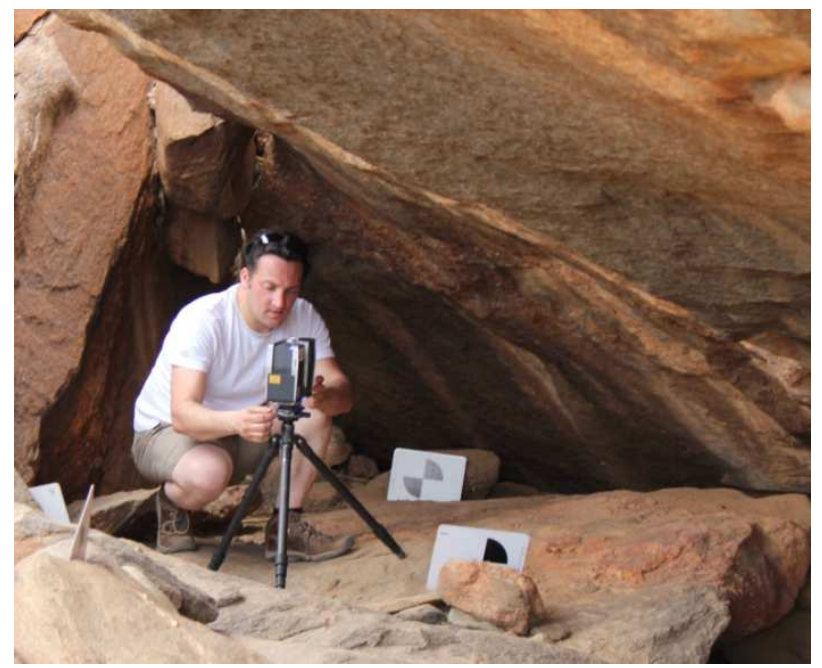

3D-laserscanning in shelter nb 2b. (Photo: V.Jallot)

In order to achieve the registering of all the scan point clouds, plastic spheres and targets have been regularly spread around the shelters. These markers, were used to provide an approached assembly, allowing a better registration in a second time using best-fit algorithms. Once assembled, the referencing of each shelter in the global coordinate system was carried out using the natural survey points. Indeed, georeferencing data being made on groups of pre-assembled scans, slightly lower accuracy of the absolute transformation did not affect the relative accuracy inside each scan cluster. Moreover, the acquisition of intensity values for each point of the cloud facilitated the picking of natural points using not only the geometry, but also the object texture, even in smooth areas provided that they are textured.

\subsection{Photogrammetric acquisitions} main steps:

Photogrammetric campaign was conducted in three

- General photos of the shelters in order to provide a global documentation of the main volumes, and to get an exploitable databank for a rough 3D reconstruction including surrounding areas.
- Detailed photogrammetric campaign for an accurate 3D reconstruction of parietal areas with millimeter precision and centimeter resolution. This is the most important operation, which was to carry out a campaign of close-up shots respecting the usual rules of photogrammetry and a specific operating mode for accurate color reproduction.

- High accuracy photogrammetric campaign on the most important parietal areas in order to provide sub-millimetric high-density 3D-models.

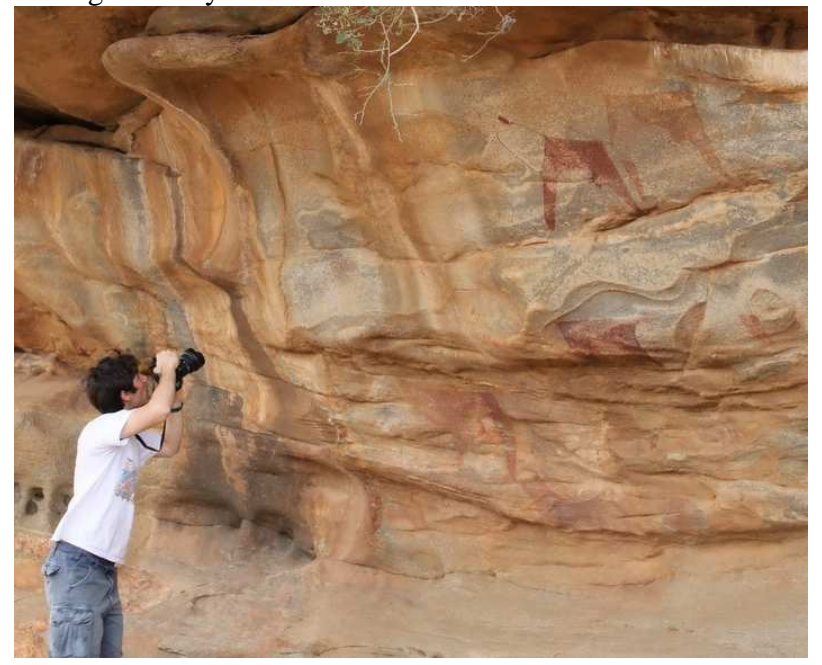

Photogrammetric acquisitions in shelter nb.2 (Photo: $\mathrm{P}$ Antoniotti)

\subsection{Topographic survey}

In order to express all the results in a same coordinate system, a series of survey points based on a polygonal canvas of ground control points was set up.

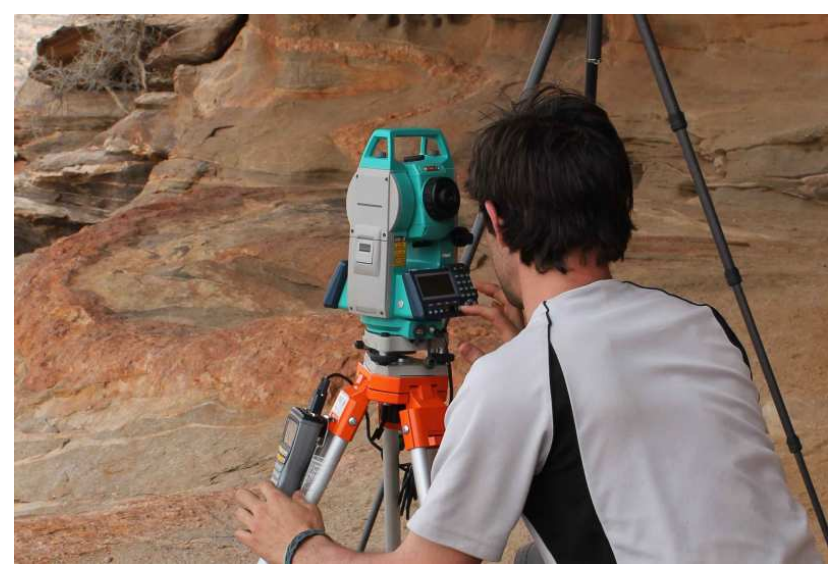

Topographic survey in shelter nb.2 (Photo: P. Antoniotti)

Given the context of the study and the geographical situation of Laas Geel, georeferencing operations were limited to ensure spatial data assembly in a local coordinate system. Indeed, the connection to an existing datum (planimetric or altimetric), would require the use a GPS positioning, involving the deployment of a much heavier instrumentation, which would then become disproportionate to the initial requirements. Additionaly, the accuracy requirements expressed in the specifications were about $1 \mathrm{~mm}$ in relative only. For this reason, we chose to use natural control points instead of survey targets. Indeed, despite the gain in precision they would have brought, 
they would also have masked some parts of the study areas, affecting the graphic quality of the final result.

\section{DATA PROCESSING}

\subsection{Laserscanning point clouds registering}

The first step was to register the lasergrammetric data. This operation, carried out in real time in order to control the process before leaving Laas Geel, allowed us to immediately dispose of a 3D exhaustive print of each shelter. The merged point clouds thus generated were afterward set in the global coordinate system, using the survey points. In order to not degrade the relative accuracy of the registration in each shelter, the transformation was performed on each of them considered as a rigid object, and not as a set of independent scan stations.

Once this first operation was completed, point clouds were processed to be usable: cleaning (removal of useless areas), noise filtering and spatial resampling with one point every 3 $\mathrm{mm}$.

\subsection{D-models generations.}

This step, which was the most important, enabled us to reveal the accurate geometry of the shelters.

The first work was to calibrate the optics to modelize their internal geometry as accurately as possible. For this, a reference pattern was used to define the calibration parameters of the cameras (according to the Brown model). The next step was the relative orientation of the photos relative to each other. This task, previously very tedious, was facilitated by the use of SIFT algorithms, which allow the automatic detection of connection points between the photos. This method works particularly well on objects widely and irregularly textured like in Laas Geel. Thus, an important number of photos were automatically processed in a few minutes, with an increased accuracy thanks to extremely redundant measures (up to 40,000 connection points per image)

The mesh creation can then start. To do so, the latest algorithms of automatic reconstruction were used, allowing to perform epipolar correlation calculations on very important sets of images, and to generate high-quality 3d-meshes semiautomatically, especially if the photos have been recorded with a sensor like the D800 (36 Mpix). For example, this sensor was most of the time associated with a $24 \mathrm{~mm}$ focal length, corresponding to an angular resolution of $12 \mathrm{mg}$, horizontally and vertically.

The generated 3D models were then processed to be usable: cleaning, removing spikes and aberrant summits, filling holes to obtain a regular and optimized triangulation. The models were then decimated selectively to reduce the data in areas with low information density, while conserving the small reliefs. The models are finally reintegrated into the $3 \mathrm{D}$ scene to be textured.

\subsection{Texturing and optimisation}

As we detailed above, the different pictures used in the 3D scene were taken with fixed parameters (time of exposure, diaphragm opening, ISO and white balance settings), not only to facilitate the automatic detection of SIFT points, but also to minimize the color gaps between photos. However, a preparatory treatment was required before implementing the texturing step itself.
First, the white balance is adjusted for each set of images, using a white target. This operation was achieved to ensure an exact rendering of colors, and thus overcome the colorimetric errors generated not only by the camera sensor, but also by the monitor calibration.

In a second time, a correction of vignetting (darkening of the corners) was applied, combined with a slight exposure adjustment, to enhance the tiny details of each image. All these operations were performed on the raw image format (*.RAW), after the relative orientation and the surface generation. Once these treatments were finished, the texturing step could begin.

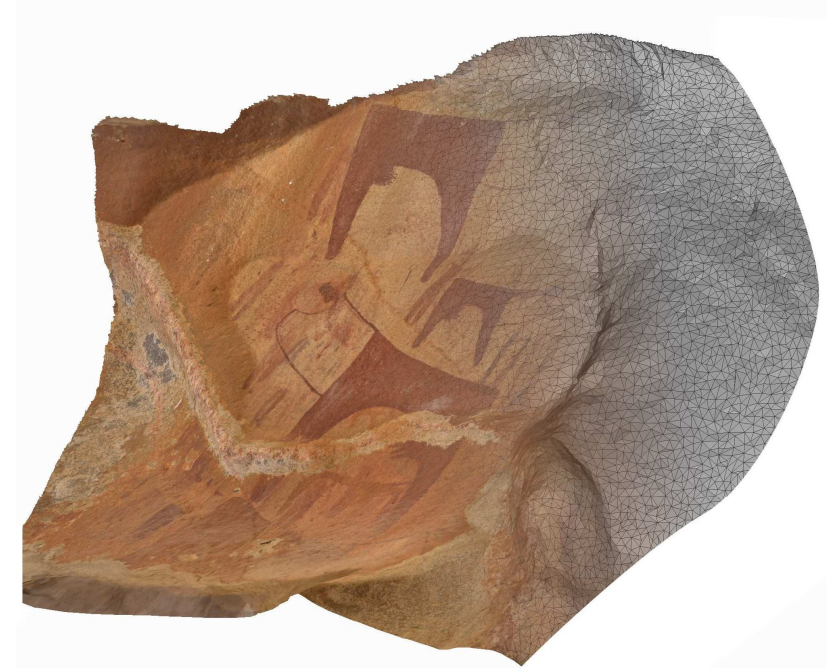

Hybrid view of shelter N.3 (detail), with texture (left) and in wireframe mode (right).

This was done semi-automatically, but a good photo quality and a judicious choice of photographs used for the projection of textures are essential for obtaining good results (avoiding mosaic imperfections and "pixel floods", consequences of a projection axis too far from the surface normal).

Once the automatic texture projection is finished, a final optimization is carried out, in order to eliminate residual defects in texture. For this, the positions and orientations of the cameras were recovered in specific software to manually control the texture projection in problematic areas.

\subsection{Scene Georeferencing}

Georeferencing phase was implemented at the end of the workflow, allowing us to use texture when picking 2D-3D correspondences. The method was the same as for lasergrammetric data and results in the final model. Given the redundancy of information between photogrammetric and lasergrammetric models, and the absence of correlation between them by the use of external survey points, a relevant control method was provided. Two analysis have been carried out, respectively on shelter 1 and 2, and the results in both cases revealed a less than $1 \mathrm{~cm}$ distance between the lasergrammetric and photogrammetric models on $90 \%$ of the surface. These results were satisfying, given the point clouds registering relative accuracy (between 5 and $7 \mathrm{~mm}$ ). 


\subsection{Sub-millimetric 3D-models generation}

The most interesting parts of the shelters, concerned by a particular archaeological study, have been recorded with a sub-millimetric resolution. To do so, an additional photogrammetric acquisition was achieved at a closer distance, in order to reveal the finest reliefs, and provide interpretation and enhanced tools for archaeologists. Among these areas, an interesting rock carving close to shelter $n .11$ can be mentioned, representing a large-horns animal.

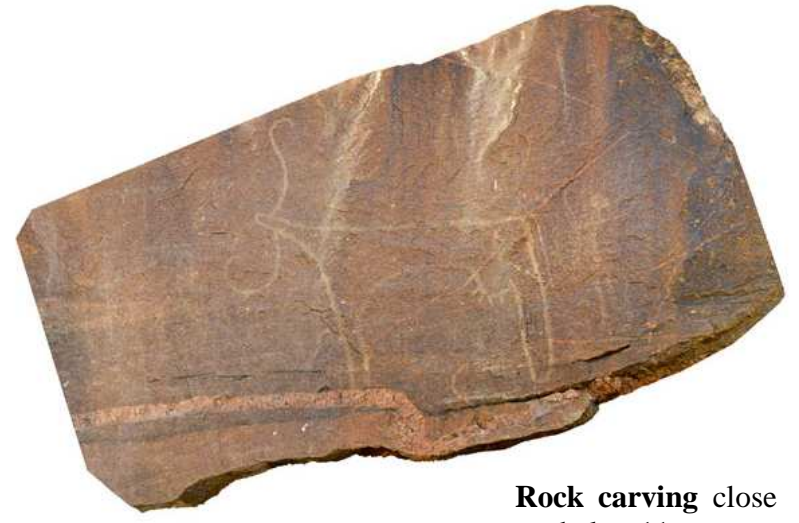
to shelter 11

This area, first integrated in the point cloud of shelter n.11, could not reveal all its details using only the lasergrammetric data, the engraving having a depth below the accuracy of the scanner (about one millimeter). To solve this problem, we decided to use very close-range photogrammetry and thus reveal the micro-details.

\subsection{Merging results in a hybrid final model}

The integration of these results in a hybrid model, allowed to insert photogrammetric high density meshes in the general models created for each shelter. The photomodels were first scaled and aligned with the global model using a best-fit registration. Both models were then merged, resulting in a hybrid model with variable density, depending on the interest of each area.

\section{EXPLOITATION}

- Ortho-photos: The final 3D-models can be used to produce ortho-photos along different axes. Two ortho-photos were produced for each shelter, including elevation (front view) and a bottom view. Depending on the volume configuration, the generation of oblique-axis orthophotos (45 degrees) was also required in some cases. The picture resolution was set to $2 \mathrm{~mm} /$ pixel, in order to allow a 1:20-scale print.

- Decimations: In order to make the results available to any type of computer power, a set of sub-models, inherited from the original models, were created at different resolutions, as much for the mesh density (number of faces) as for the texture UV-map resolution. This step also ensured the dissemination of results on any type of digital media.

$\downarrow$ Below: Ortho-image (bottom view) of shelter N.1

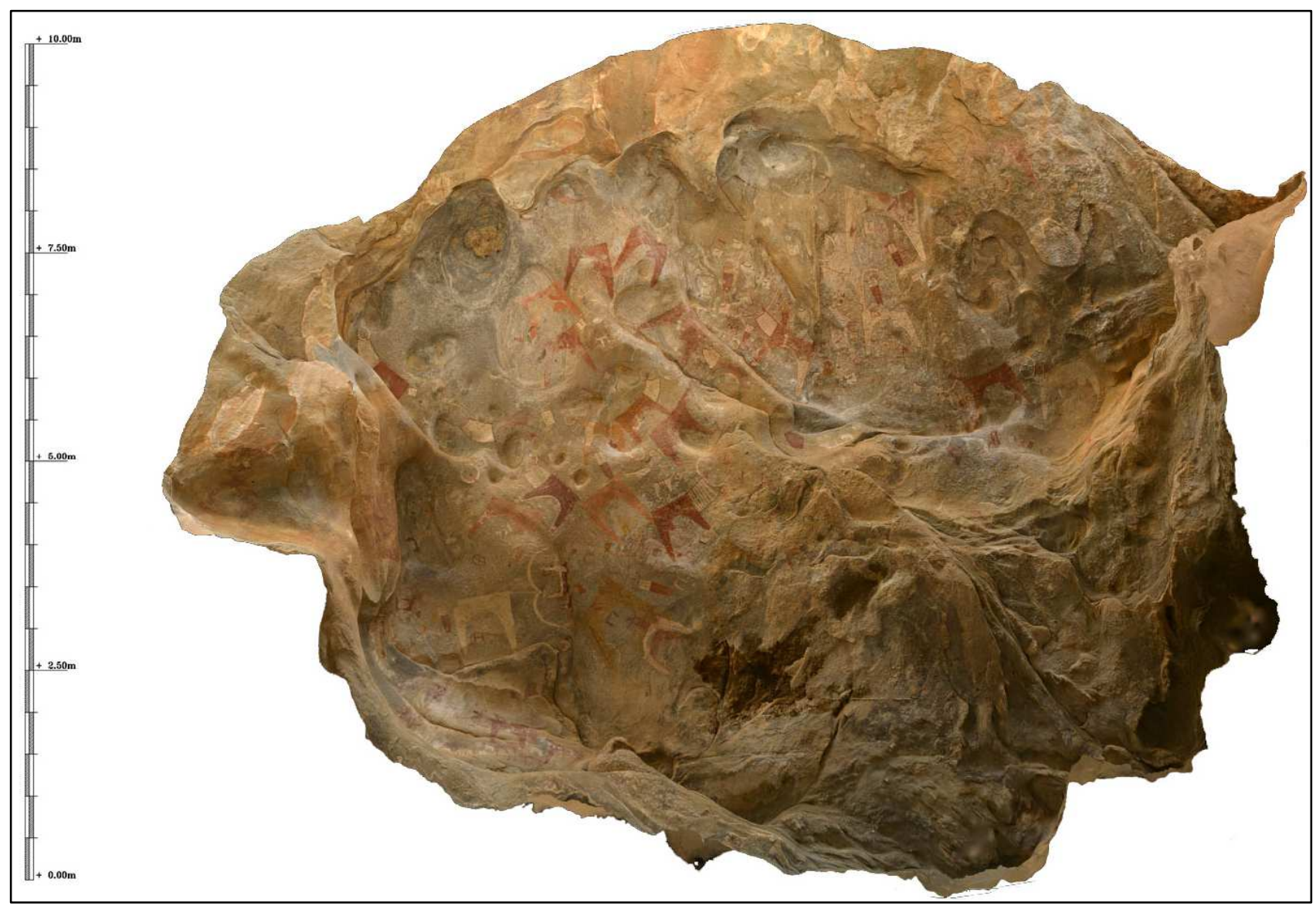




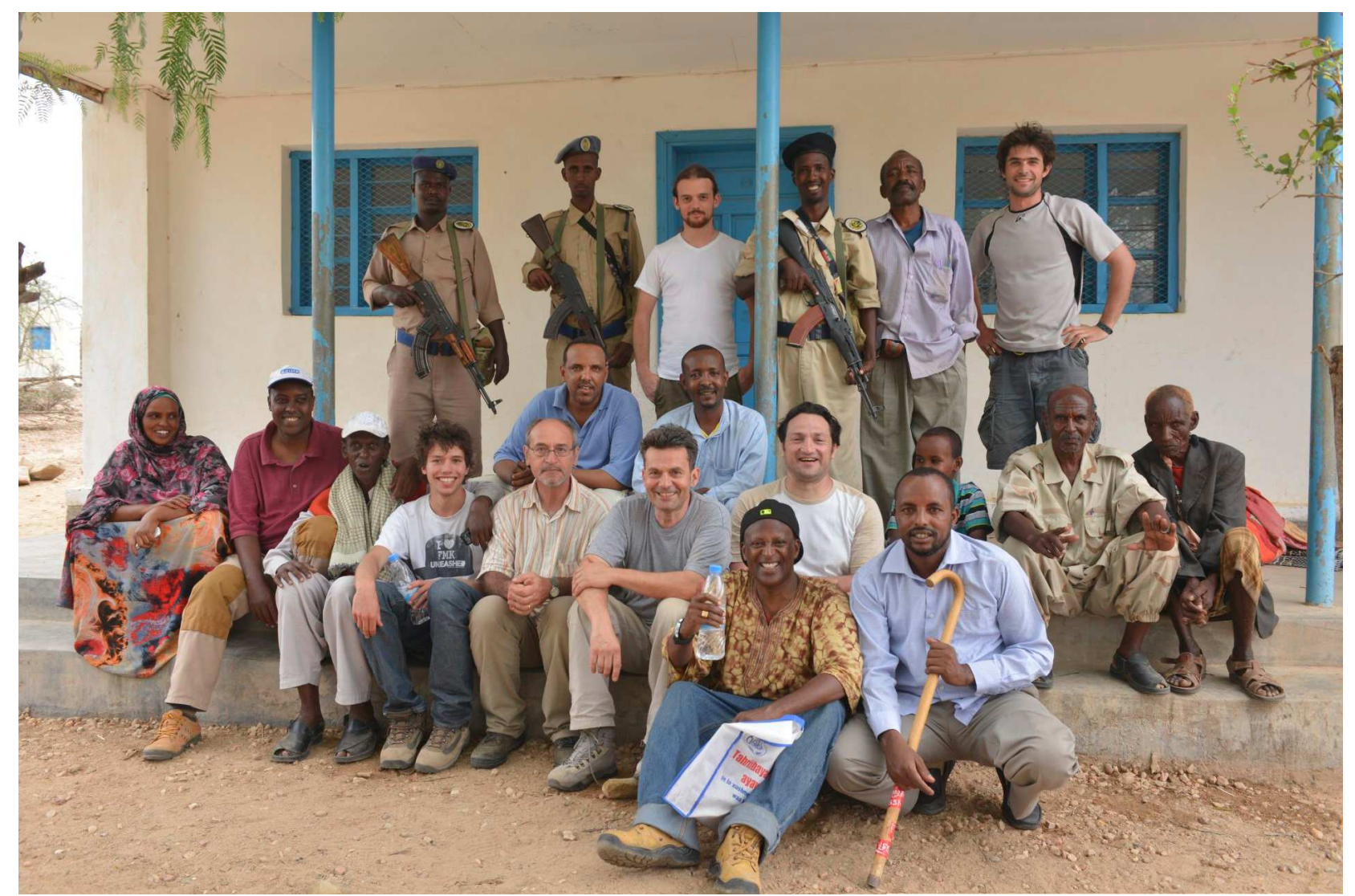

The whole team in front of the Museum

- Export models in various formats. Finally, to ensure the readability of the results in different working environments, whatever the software used, several export formats have been provided.

These possibilities offered by the models thus generated also allow to consider several other types of exploitations according to the needs expressed. Mention may be made among them the production of animations, virtual tours and augmented reality applications.

\section{CONCLUSION}

This mission successfully completed in June 2012, reached the objectives set by our clients, who were focused on three main axes: The capture of an exhaustive digital footprint of the shelters for the purpose of conservation on the one hand, the production of high quality documentation for the operation of the scanning, not only to provide analytical tools for archaeologists, but also vectors of communication of results for all users, and the enhancement of the partnership already established with local authorities to put the site in relief and encourage its protection.

Within Art Graphique et Patrimoine, finally, this adventure was the opportunity to enhance digitizing techniques used for many years by the development of innovative tools for improving results. The geographic context of the site required a very light instrumentation and a guarantee of results without coming back on the site. We also had to adopt a particularly rigorous methodology when planning operations, and when processing data in real time during the mission.

Many other remarkable sites exist in Somaliland, and the success of this first mission paves the way for other documentation projects. Located in remote areas, they have not been studied yet, and their documentation is undoubtedly a leading perspective in the field of archaeological documentation of the Horn of Africa.

\section{References from websites:}

La Croix [fr]:, 5.06.2012 :

http://www.la-croix.com/Actualite/Monde/Des-peinturesvieilles-de-5-000-ans-decouvertes-en-Somalie-_NG_-2012-06$\underline{05-814681}$

French Embassy in Djibouti [fr]:, 06.2012:

http://www.latitudefrance.org/Une-mission-archeologiqueexceptionnelle-au-Somaliland.html

FARO case studies [fr]:, 03.04.2013

http://www.faro.com/measurementsolutions/industries/heritage/2013/04/03/the-cave-paintings-of$\underline{\text { laas-geel }}$

Wikipedia page on Laas Geel [fr]: http://fr.wikipedia.org/wiki/Laas_Geel 